\title{
Fabrication of Breathable Film Using Diffractive Multi-beam Nanosecond Laser Patterning of Polypropylene Film
}

\author{
Dongyoon Yoo*, Hun-Kook Choi ${ }^{*}$, Jae Hee Sung ${ }^{*}$, Ik-Bu Sohn $^{*}$, Jongyeol Kim ${ }^{* * * * *}$,Young-Han Kim ${ }^{* * * *}$, \\ and Md. Shamim Ahsan ${ }^{* * * * *}$ \\ *Advanced Photonics Research Institute (APRI), Gwangju Institute of Science and Technology \\ (GIST), 123 Cheomdan-gwagiro, Buk-gu, Gwangju 61005, Republic of Korea \\ E-mail: ibson@gist.ac.kr \\ ${ }^{* *}$ Korea Atomic Energy Research Institute (KAERI), 989-111, Yuseong-gu, Daejeon 34057, \\ Republic of Korea \\ ${ }^{* * *}$ Department of Electrical and Electronic Engineering, Hanyang University, 222 Wangsimni-ro, \\ Seongdong-gu, Seoul 04763,Republic of Korea \\ **** Daerung Packaging Industry, Sundong-ri 400, Chowol-eup, Gwangju City, Gyeongi-do 464-864, \\ Republic of Korea \\ ${ }^{* * * * *}$ Electronics and Communication Engineering Discipline, Science Engineering and Technology \\ School, Khulna University, Khulna 9208, Bangladesh \\ E-mail: shamim@kaist.ac.kr
}

\begin{abstract}
This paper demonstrates the manufacturing of breathable film by patterning micro-holes on the surface of oriented polypropylene films by means of diffractive multi-beam nanosecond laser assisted micro-machining technology. Using a $1 \times 3$ diffractive beam splitter, we have split a single laser beam into three parallel beams and have irradiated them sequentially in a row at a constant speed to overlap three pulses in each irradiated spot of the film surface. As a consequence, microholes' array of high depth and wide diameter is formed on the film surface. We also fabricated breathable polypropylene films by single laser beam engraved micro-holes' array. Multi-pulse engraved micro-holes show better aspect ratio, compared to single pulse engraved micro-holes. To study the influence of average pulse energy on breathability of the films, we have fabricated microholes array on the polypropylene film surface by varying the average pulse energy. Furthermore, we investigate the oxygen transfer rate through the multi-pulse irradiated breathable films. The experimental results exemplify that, oxygen transfer rate increases with the number of irradiated laser pulses in each spot of the oriented polypropylene films. The fabricated breathable films will pave the way for technological advancement, especially in packaging, and manufacturing sanitary pad, diaper and water proof vinyl.
\end{abstract}

DOI: $10.2961 / \mathrm{jlmn} .2020 .02 .2005$

Keywords: nanosecond laser, micro-holes' array, oriented polypropylene (OPP) film, breathable film, oxygen transfer rate (OTR)

\section{Introduction}

Nowadays, breathable films have wide range of applications in a diverse field of science and technology, especially in the packaging industry. Breathable films have the characteristics of allowing air flow through the film. During last several decades, oriented polypropylene films have been widely used in the packaging industry as breathable films because of their excellent thermal stability, chemical resistance, and physiological compatibility [1-5]. The micro-perforated polypropylene films are able to breathe, which property makes them suitable for packaging biochemically active agricultural products including fresh fruits, vegetables, herbs, for disposable hygiene articles, and for protective health care garments where air breathability is a key requirement [3]. The formation of micropatterns, especially micro-holes and micro-porous on the surface of OPP films causes the increase in flow of oxygen and carbon dioxide through the polypropylene container.

There have been reports on the formation of breathable films by micro-structuring of OPP films using various micro-machining technologies [1-5]. P. C. Wu et al. have analyzed the breathability of various micro-porous films with variable composition by investigating the oxygen transfer rate and moisture vapor transmission rate [1]. A novel technique of fabricating waterproof breathable composite materials has been proposed by another research group [2]. Compared to other processing technologies, lasers have shown great promises to produce good quality breathing films [3-5]. Dr. Sohn and his research group have reported the formation of breathable films by printing micro-holes' array on the surface of oriented polypropylene films by means of single-beam laser writing technology using different laser systems including nanosecond, picosecond, and femtosecond lasers $[3,4]$. The same research group utilized a single $\mathrm{CO}_{2}$ laser beam to pattern micro-holes on the surface of OPP films functioning as breathable films. $\mathrm{CO}_{2}$ laser assisted micro-structured OPP films showed immense breathability and showed promises in packaging fresh veg- 
etables and fruits [5]. Z. Wang et al. reported the microperforation of thin biomimetic films by means of singlebeam femtosecond laser writing technique [6]. Although laser assisted technologies show good results in fabricating quality breathable films in small scale, most of the technologies suffer from poor throughput when commercial production is considered. Besides, there are scopes of improving the breathing rate of the films and quality of the microstructures. Thus, more investigations are desirable to find a novel technique of manufacturing good quality breathable films having high breathing rate and throughput, suitable for commercial production.

For simultaneous micro/nano-patterning of a large sample area, researchers proposed two different kinds of laser assisted patterning technologies: particle lens array (PLA) incorporated technology [7-10] and diffractive optical element (DOE) integrated technology [11-13]. PLA assisted laser processing technology utilizes the near-field enhancement induced by the micro/nano-metric spherical particles or lenses, which are closely distributed on the sample surface. Each particle or lens fabricates a small hole on the material surface and consequently, simultaneous formation of nano-holes on the sample surface has been achieved in a large variety of materials including silicon [7,8], glass [9], and photo-polymerized resin [10]. However, nano-holes might not be enough for breathability of the films when the film thickness is in the range of several micrometers. As a result, this technology might not be adequate enough to serve our purpose, i.e., producing good quality breathing films with high throughput. Diffractive multiple beams have been utilized by several research groups to improve the processing time by means of simultaneous fabrication of micro-structures on the surface of various materials including titanium alloy (Ti6Al4V), glass, SU-8 photoresist, and silicon [11-17]. Two different kinds of DOEs are widely utilized to split the incident laser beam into several diffractive laser beams: spatial light modulator (SLM) [11-13] and diffractive beam splitter (DBS) [14-17]. Both of these DOEs are suitable for simultaneous patterning of micro/nano-holes on material surface. However, a single time exposure of laser beam on material surface can produce low depth holes and may not create enough openings in the samples for breathing. R. Zhou et al. demonstrated the enhancement of laser ablation by spatial doublepulse interaction effect where they utilized DBS to split a single laser beam into two beams [17]. However, interaction of laser pulses varies with materials. On the contrary, application of multi-beam technology for forming microlens array on a large area, especially in fabricating breathable films deserves more research. Furthermore, the impact of triple or higher number of split laser beams on the enhancement of laser ablation and their interaction with the OPP film need further investigations. Therefore, discovery of a fast and flexible technique for fabricating breathable films comes to the forefront of the research field.

In this paper, we proposed a novel technique of forming breathable OPP films by irradiating both single and multiple laser pulses in each irradiated spot. Single laser pulses were irradiated by a single laser beam. The nanosecond laser beam was split into three beams using a $1 \times 3$ DBS and sequentially scanned on the OPP films' surface to irradiate three laser pulses in each spot, which in turn engraved pe- riodic micro-holes of wide diameter and high depth on the OPP films. The depth and diameter of the holes showed increasing trend with the increase of the number of applied laser pulses in each spot and the pulse energy. Multi-pulse irradiated micro-holes showed better aspect ratio (width to depth), compared to single pulse engraved micro-holes. The width and depth of the micro-holes had significant influence on the OTR of the OPP films. More importantly, the processing time was reduced significantly, which in turn increased the overall throughput. The micro-patterned OPP films showed immense breathing capability and showed great potential for commercial applications.

\section{Experimental Details}

We carried out the experiments by means of an ultraviolet pulsed nanosecond laser source (AVIA 266-3, Coherent), operating at the central wavelength of $266 \mathrm{~nm}$. The laser beam irradiated $20 \mathrm{~ns}$ laser pulses at a pulse repetition rate of $30 \mathrm{kHz}$. To split the incident laser beam into three parallel beams, we guided the nanosecond laser beam by means of several mirrors and passed the beam through a $1 \times 3$ diffractive beam splitter where the split angle of the beam splitter was $0.32^{\circ}$. Before the DBS, the beam diameter was $\sim 2 \mathrm{~mm}$. However, the beam diameter of the diffracted laser beams was about $10 \mu \mathrm{m}$ after the DBS. The collimated laser beams were focused on the OPP film surfaces by utilizing a convex lens (focal length: $57.3 \mathrm{~mm}$ ). The spatial distance between the split laser beams at the focal point was $\sim 300 \mu \mathrm{m}$. To pattern micro-holes array on the OPP films' surface, these multiple laser beams were focused on the film surface and the translation stage was moved at a scanning speed of $10 \mathrm{~mm} / \mathrm{s}$. As a consequence, the beams moved $300 \mu \mathrm{m}$ in each step from their previous position and irradiated one pulse. Consequently, micro-holes were fabricated $300 \mu \mathrm{m}$ apart. In contrast, the vertical scanning step of the laser beams was $300 \mu \mathrm{m}$. Fig. 1(a) illustrates the schematic diagram of the experimental setup.

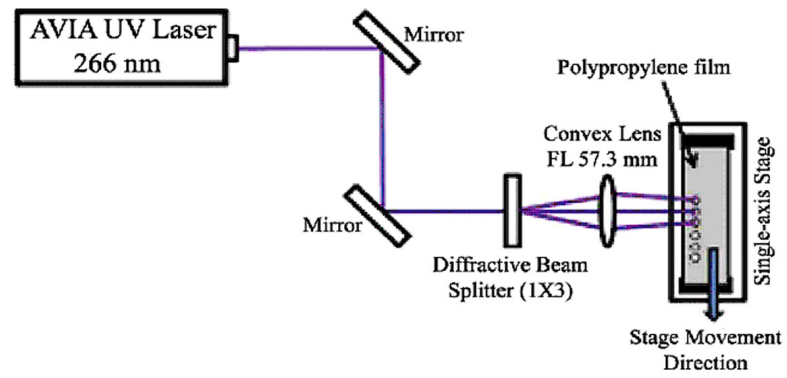

(a)

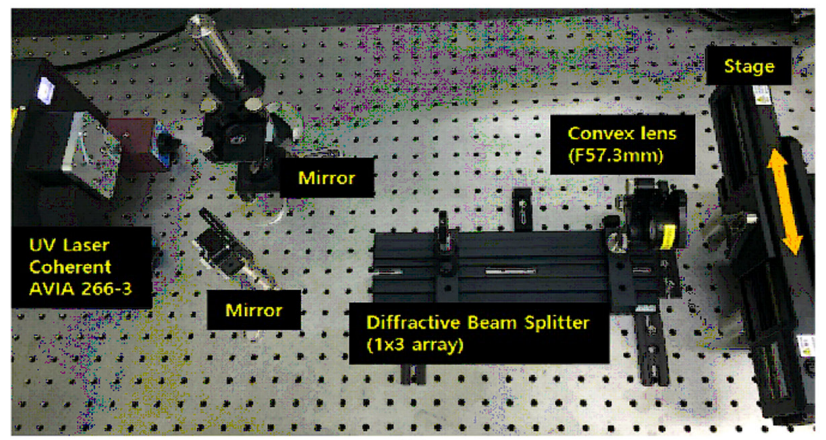

(b)

Fig. 1 Experimental setup of the ultraviolet pulsed nanosecond laser system. (a) Schematic diagram; (b) Photograph. 
The photograph of the ultraviolet pulsed nanosecond laser system is illustrated in Fig. 1(b). Oriented polypropylene films of $30 \mu \mathrm{m}$ thickness were placed on a single axis step motorized stage (SGSP-150, $80 \mathrm{~mm}$ stroke), as shown in Fig. 1(b). To fabricate periodic micro-holes' array on the surface of OPP film, the laser beam with an average pulse energy $(E)$ varying from $7-50 \mu \mathrm{J}$ was irradiated on the sample surface. As a consequence, the OPP films turned into breathable films. Using both the single-beam and multi-beam nanosecond laser based technologies, we fabricated total twenty $(10 \times 2)$ breathable OPP films. After laser processing, the micro-structured samples went through ultrasonic cleaning in deionized water for approximately 30 minutes. The micro-holes engraved samples were investigated under an optical microscope (ZEISS, Axioskop 40). We also measured the oxygen transfer rate through the periodic micro-holes' engraved OPP films to investigate the breathability of the films.

\section{Experimental Results}

By irradiating nanosecond laser pulses, we fabricated breathable films by patterning periodic micro-holes' array on the surface of OPP films. The micro-holes were fabri- cated by applying a single pulse and 3 pulses in each irradiated spot of the OPP films. Initially, single laser pulses with pulse energy varying from 33-50 $\mu \mathrm{J}$ were irradiated over a large sample area to fabricate micro-holes' array on OPP films' surface. When the average pulse energy was 33 $\mu \mathrm{J}$, consistent micro-holes with diameter of approximately $33 \mu \mathrm{m}$ were observed on the surface of most of the OPP film samples, the optical microscope (OM) image of which is illustrated in Fig. 2(a). With the increase of the pulse energy, the diameter and depth of the micro-holes were increased. At a pulse energy of $40 \mu \mathrm{J}$, the diameter of the micro-holes reached to $43 \mu \mathrm{m}$, as shown in the OM image of Fig. 2(b). When the pulse energy was $50 \mu \mathrm{J}$, micro-holes with diameter of $49 \mu \mathrm{m}$ was evident on the surface of OPP film, as depicted in Fig. 2(c). In all these cases, we observed heat affected zones around the micro-holes, as depicted in Figs. 2(a-c). The optical microscope image of the micro-holes' array fabricated at $50 \mu \mathrm{J}$ energy is illustrated in Fig. 2(d). The experimental results show that, aspect ratio of the single pulse irradiated micro-holes decreases with the increase of the pulse energy. Excellent aspect ratio was achieved at a pulse energy of $50 \mu \mathrm{J}$. Fig. 2(e) plots the width and depth of the micro-holes' with respect to the

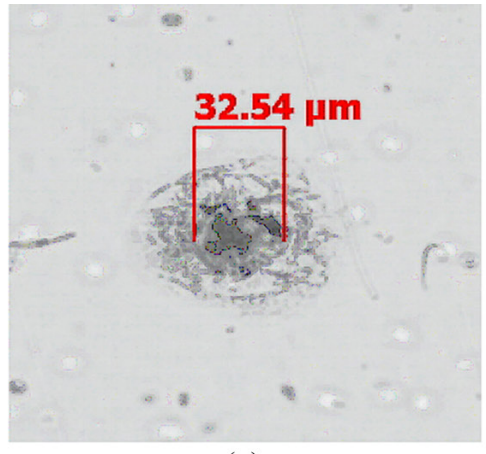

(a)

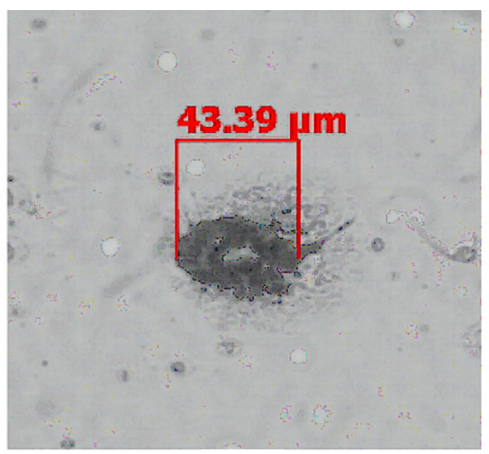

(b)

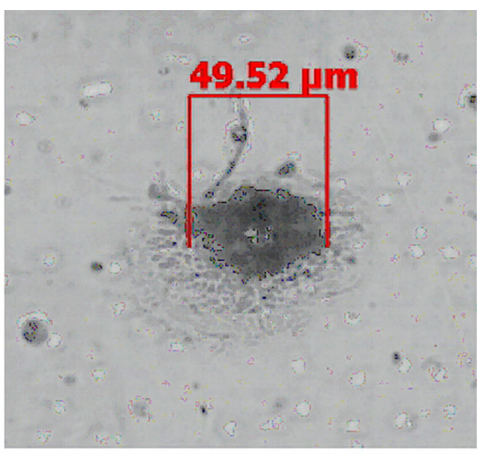

(c)

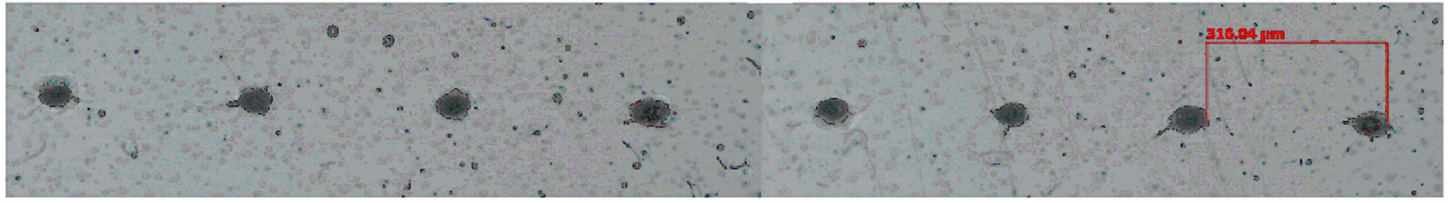

(d)

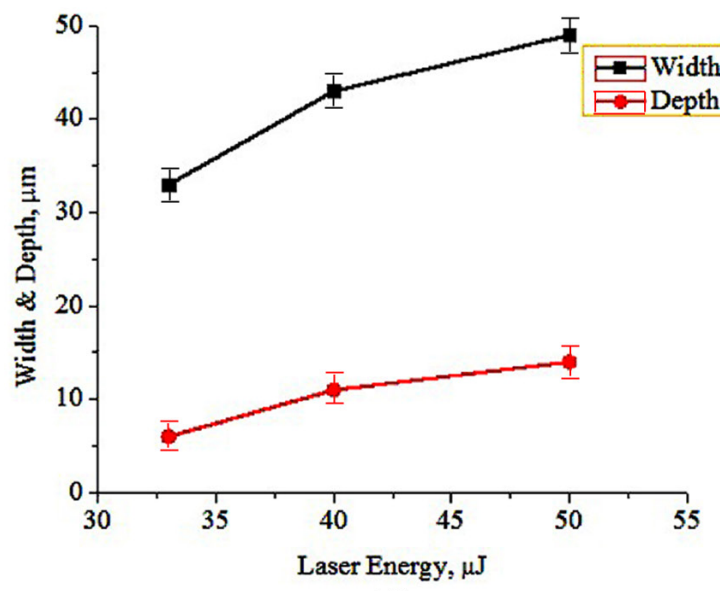

(e)

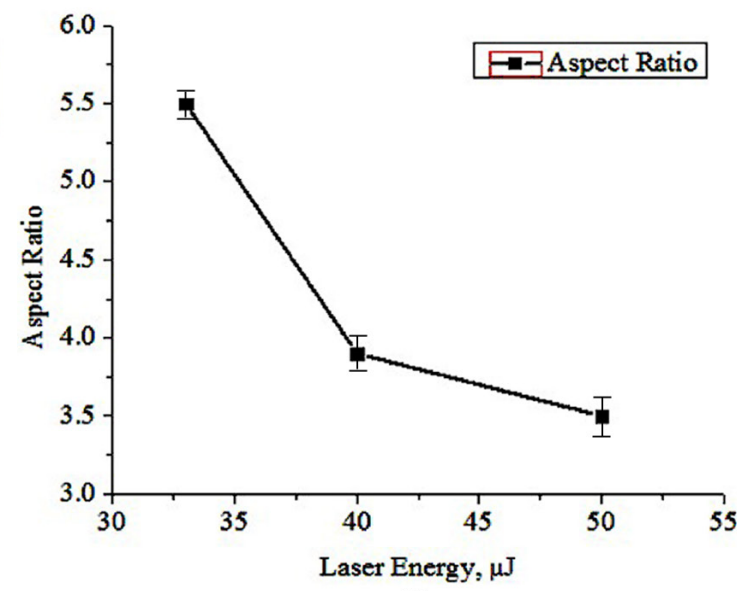

(f)

Fig. 2 Single laser pulse assisted fabrication of micro-holes on OPP film surface by varying the average pulse energy energy from 33-50 $\mu \mathrm{J}$. (a-c) OM images of the micro-holes: (a) $E=33 \mu \mathrm{J}$, (b) $E=40 \mu \mathrm{J}$, (c) $E=50 \mu \mathrm{J}$; (d) OM image of the Micro-holes' array at $E=50 \mu \mathrm{J}$; (e) Width and height of the micro-holes fabricated with different $E$; (f) Aspect ratio of the single pulse irradiated micro-holes. The variations of the physical parameters of the micro-holes are indicated by the bars of the Figs. 2(e) and 2(f). 
pulse energy of the irradiated pulses. The aspect ratio of the single pulse irradiated micro-holes is illustrated in Fig. 2(f). The bars of the Figs. 2(e) and 2(f) represent the variation of the physical parameters of the micro-holes. The width, depth, and aspect ratio of the micro-holes, observed in most of the samples, fabricated using single pulse irradiation are summarized in Table 1.

Table 1 Width, depth, and aspect ratio of the single pulse engraved micro-holes on OPP films

\begin{tabular}{cccc}
\hline \multirow{2}{*}{ Entity } & \multicolumn{3}{c}{ Pulse Energy, $\mu \mathrm{J}$} \\
\cline { 2 - 4 } & 33 & 40 & 50 \\
\hline Width, $\mu \mathrm{m}$ & 33 & 43 & 49 \\
Depth, $\mu \mathrm{m}$ & 6 & 11 & 14 \\
Aspect Ratio & 5.5 & 3.9 & 3.5 \\
\hline
\end{tabular}

In order to investigate the impact of three laser pulses on the formation of the micro-holes and to examine the breathability of these micro-holes array engraved breathable OPP films, we applied three laser pulses in each irradiated spot by sequential movement of the multi-beam laser at a constant speed of $10 \mathrm{~mm} / \mathrm{s}$. Average pulse energy of the laser beams was varied from 7-17 $\mu \mathrm{J}$. Schematic diagram of the proposed sequential patterning concept is represented in Fig. 3. The numerical values in each figure of Figs. 3(a-d) indicate the number of irradiated laser pulses in a particular spot. The focal points of the parallel laser beam coming out from the $1 \times 3$ diffractive beam splitter was aligned in a horizontal line. After first time exposure of the laser beam, the three parallel beams strike at three different locations, each of which was $300 \mu \mathrm{m}$ apart. Since the focal points of the parallel laser beams were aligned properly, micro-holes were evolved in each irradiated spot forming a horizontal line, as shown in Fig. 3(a). When the parallel beams were moved by one step, the first beam was moved to the position of the second beam of Fig. 3(a) and the second beam was moved to the position of the third beam of Fig. 3(a). As a consequence, two pulses were irradiated in these two spots. On the contrary, the third beam was moved to a new position, as represented in Fig. 3(b).

After second step movement of the laser beams, the first and second beams of Fig. 3(a) now moved to the third and fourth position (as shown in Fig. 3(c)) and the third beam of Fig. 3(b) was moved to a new position. Due to the sequential movement of the laser beams, three pulses were irradiated in each irradiated spots after the third step movement except the first two and last two positions, as illustrated in Fig. 3(d). The number of overlapped laser pulses in each irradiated spot is listed in Fig. 3 for each step. Fig. 3(e) illustrates the OM image of the micro-holes encoded OPP film after nanosecond laser irradiation with average pulse energy of $17 \mu \mathrm{J}$ before ultrasonic cleaning. The magnified OM images along with the diameter of the micro-holes are shown in the insets of Fig. 3(e). The OM image of the micro-holes array encoded OPP films after ultrasonic cleaning is illustrated in Fig. 3(f). The horizontal and vertical periods of the micro-holes were $300 \mu \mathrm{m}$.

By varying the pulse energy from 7-17 $\mu \mathrm{J}$, we fabricated periodic micro-holes of variable diameter on the surface of OPP films by applying three pulses in each irradiated

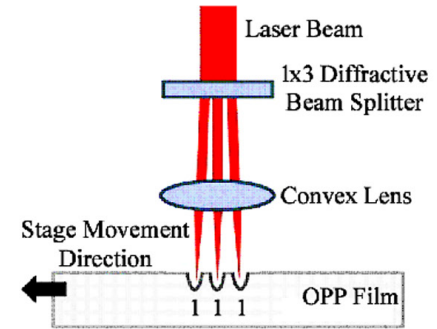

(a)

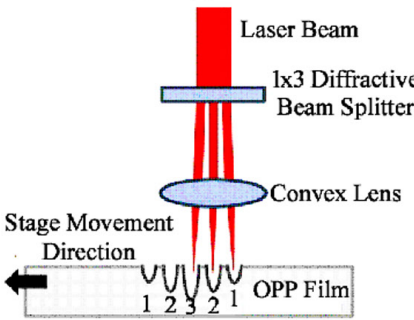

(c)

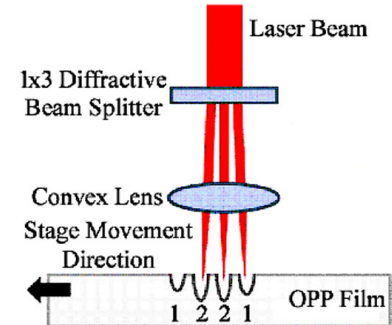

(b)

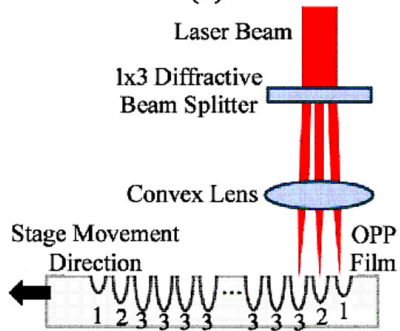

(d)

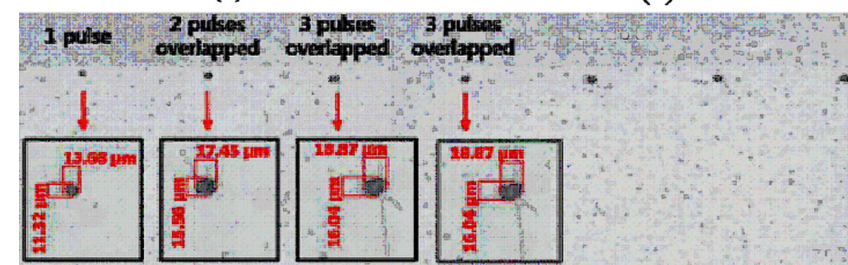

(e)

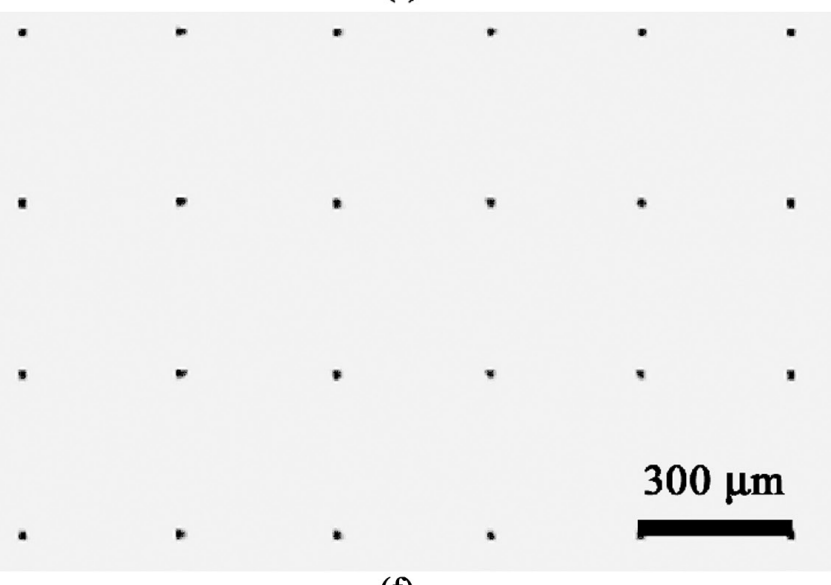

(f)

Fig. 3 Formation of micro-holes' array on OPP film by diffractive multi-beam patterning technique. (a) After first time laser irradiation; (b) After one step movement of the laser beams; (c) After two step movement of the laser beams; (d) Periodic micro-holes due to sequential movement of the laser beams; (e) OM image (top view) of Fig. 3(d) and magnified image of the micro-holes; (f) OM image of the micro-holes' array on OPP film surface after irradiation of three laser pulses of $17 \mu \mathrm{J}$ pulse energy in each irradiated spot.

spot, optical microscope images of which are depicted in Figs. 4(a-d). When three laser pulses with pulse energy of 7 $\mu \mathrm{J}$ were applied one after another in a single spot, microholes of $12 \mu \mathrm{m}$ diameter were evolved on the OPP film surface, as represented in Fig. 4(a). The same process is repeated by changing the energy of the laser pulses to $11 \mu \mathrm{J}$, $13 \mu \mathrm{J}$, and $17 \mu \mathrm{J}$ and consequently, micro-holes array were engraved on the OPP film surface with holes' diameter i.e., width of $13 \mu \mathrm{m}, 14 \mu \mathrm{m}$, and $16 \mu \mathrm{m}$, respectively (Fig. 4(bd)). Like single pulse engraved micro-holes, heat affected zone was also evident for the multi-pulse irradiated microholes. Fig. 4(e) plots the dependence of width and depth of 


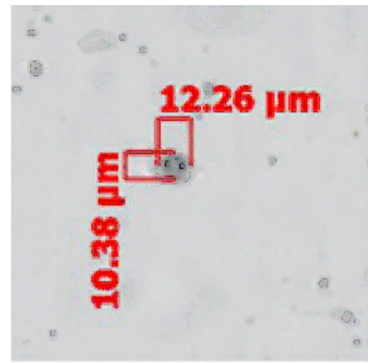

(a)

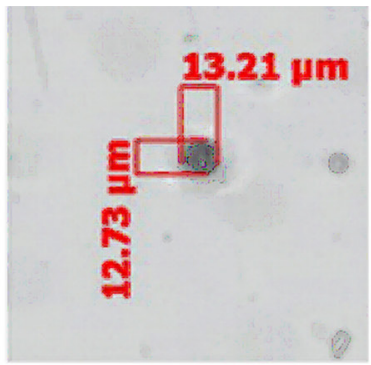

(b)

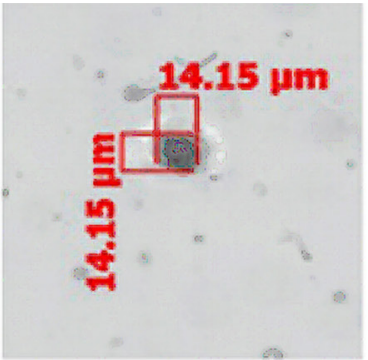

(c)

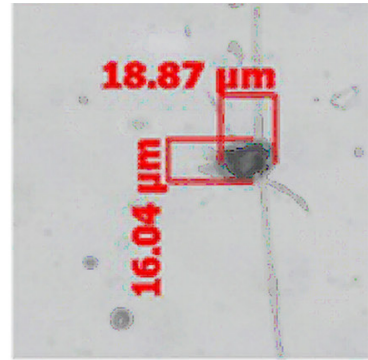

(d)

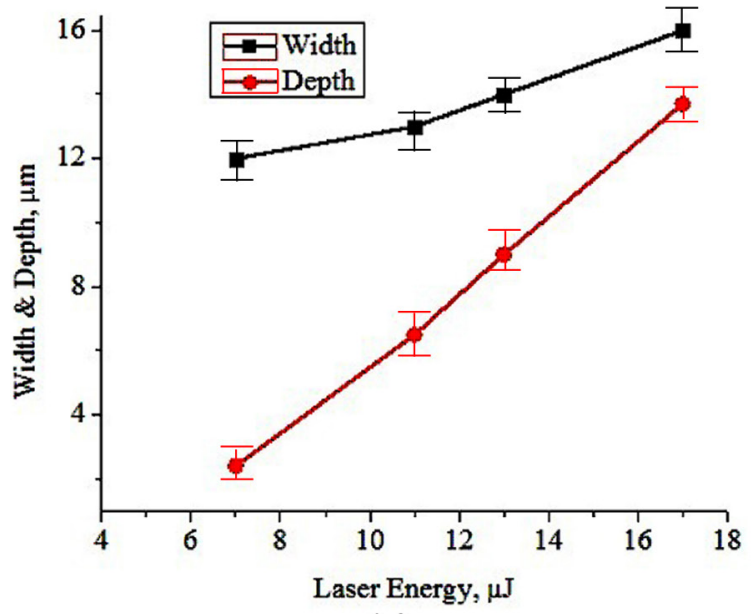

(e)

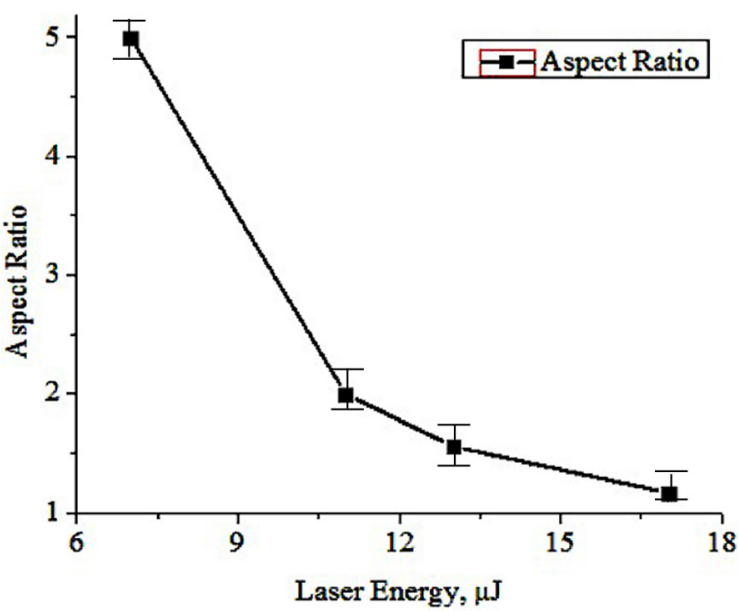

(f)

Fig. 4 Micro-holes on OPP film surface after the irradiation of three laser pulses under variable average pulse energy $(E)$. (a-d) Optical microscope images of the micro-holes: (a) At $E=7 \mu \mathrm{J}$, (b) at $E=11 \mu \mathrm{J}$, (c) At $E=13 \mu \mathrm{J}$, (d) at $E=17 \mu \mathrm{J}$; (e) Plot of width \& depth of the micro-holes with respect to pulse energy; (f) Plot of aspect ratio of the micro-holes with respect to pulse energy. The variations of the physical parameters of the micro-holes are indicated by the bars of the Figs. 4(e) and 2(f).

the micro-holes on the pulse energy, whereas Fig. 4(f) represents the relationship between the pulse energy and the aspect ratio of the micro-holes. Like single-pulse engraved micro-holes, the aspect ratio of the multi-pulse exposed micro-holes showed decreasing trend with the increase of average pulse energy. Micro-holes of excellent aspect ratio were achieved for average pulse energy of $17 \mu \mathrm{J}$. The experimental results of the three pulses irradiated OPP samples are summarized in Table 2.

Table 2 Width, depth, and aspect ratio of the three pulses irradiated micro-holes on OPP films

\begin{tabular}{ccccc}
\hline Entity & \multicolumn{4}{c}{ Pulse Energy, $\mu \mathrm{J}$} \\
\cline { 2 - 5 } & 7 & 11 & 13 & 17 \\
\hline Width, $\mu \mathrm{m}$ & 12 & 13 & 14 & 16 \\
Depth, $\mu \mathrm{m}$ & 2.4 & 6.5 & 9 & 13.7 \\
Aspect Ratio & 5 & 2 & 1.56 & 1.17 \\
\hline
\end{tabular}

To investigate the breathability of the micro-structured OPP films, we measured the OTR of the multi-pulse engraved OPP films, where the number of laser pulses in each irradiated spot was varied from 3 to 15 (in this manuscript, only 3 pulses engraved samples were presented). Fig. 5 depicts the OTR data of the micro-holes array encoded OPP films for different number of incident laser pulses, which represents breathability of the micro-holes array engraved OPP films. The experimental results confirm that, the OTR increases with the increase of the number of applied laser pulses. Due to the increase in the number of laser pulses, the width as well as depth of the micro-holes was increased. This phenomenon might have caused the increase in the OTR rate of the breathable OPP films with respect to number of irradiated laser pulses in each spot.

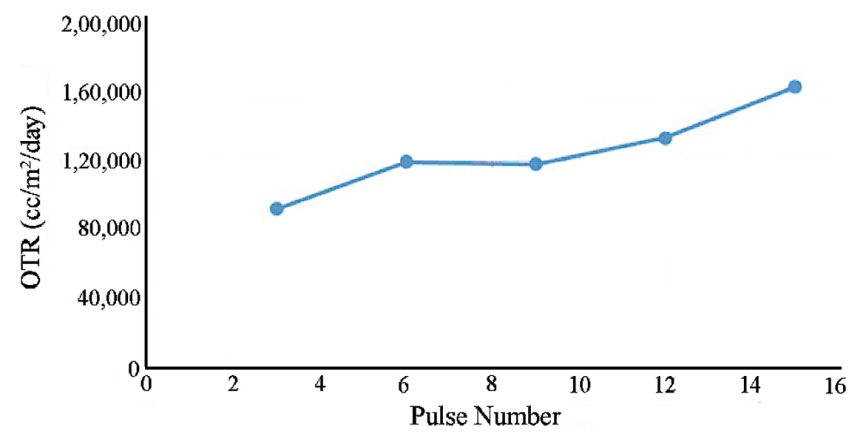

Fig. 5 Oxygen transfer rate characteristic of the breathable films fabricated by applying different number of laser pulses in each spot at average pulse energy of $17 \mu \mathrm{J}$.

\section{Discussion}

Ablation occurs in a material when the laser fluence exceeds a distinct threshold value of that material. To analyze the spot size of the ablated area, at first we considered single pulse assisted ablation of a material with ablation threshold fluence $F_{t h}$. The solution for determining the diameter $D$ of the micro-holes is as follows [20]:

$$
D=\sqrt{2 w_{0}^{2} \ln \left(F_{0} / F_{t h}\right)}=\sqrt{2 w_{0}^{2}\left[\ln \left(F_{0}\right)-\ln \left(F_{t h}\right)\right]}=\sqrt{2 w_{0}^{2} \ln \left(E_{0} / E_{t h}\right)}(1)
$$


where $w_{0}$ is the half of the beam waist size and $F_{0}$ is the peak laser fluence, $F_{t h}$ is the threshold fluence (energy per area) for a particular material, and $E_{0} \& E_{t h}$ are the peak pulse energy of the laser beam \& threshold energy of the material. When we increase the number of applied laser pulses $(N)$ from 1 to 3 , it is evident that the ablation occurs at low laser fluence, i.e. low pulse energy. The decrease in the threshold fluence for multiple pulses can be attributed as the increased absorption due to accumulated damaging effects from individual pulses. The effect of incubation on ablation threshold can be quantified by the following equation [20].

$$
F_{t h}(N)=F_{t h}(1) N^{\xi-1}
$$

where $\zeta$ is a material dependent statistical parameter. The reduction of threshold fluence required for material ablation, caused due to the exposure of multiple laser pulses, might be influenced by long time continuous thermal diffusion by laser beam [17].

The micro-holes of Figs. 2 and 4 suggest increasing trend of diameter with the increase of average pulse energy. Initially, the diameter of the holes had increased sharply with the pulse energy. Later on, the diameter increasing rate had slowed down with the increase of pulse energy. These results are in substantial agreement with the model proposed in for Gaussian laser beam [18]. The pulse energy dependence of micro-hole's diameter for Gaussian laser beam can be obtained using the equation as follows [18]:

$$
D=A \ln \sqrt{\frac{E}{E_{t h}}}
$$

where $D$ is the micro-hole's diameter and $E$ is the pulse energy. Here $E_{\text {th }}$ and $A$ are fitting parameters. In Gaussian laser beam, higher energy is evident at the center of the beam, compared to the periphery of the laser beam. When pulse energy is increased, the energy at the beam center also increased along with the energy of the beam periphery. As a consequence, the depth as well as the diameter of the micro-holes also increased, as evident from Figs. 2(e) and 4(e). Compared to single-beam engraved micro-holes reported in other research works [3-5], the proposed threebeam technique engraved micro-holes showed wider width and higher depth.

The maximum number of overlapped pulses and the average pulse energy required to fabricate breathable films are directly dependent on the thickness of the OPP films. When the film thickness is high, we require more pulses with high pulse energy to be irradiated at the same spot. There is a tradeoff between the number of required laser pulses and pulse energy. Equation (2) exemplifies that, when number of irradiated laser pulses is high, comparatively low pulse energy is enough to fabricate deep microholes on thick OPP films. In such cases, we require to split the laser beam into many consistent beams that is very difficult to achieve. Therefore, we should focus on controlling the consistency of pulse energy of the multiple beams rather than splitting the laser beam into many parallel beams.

We noticed heat affected zones around the single-beam and multi-beam engraved micro-holes (see Figs. 2(a-d), Figs. 3(e-f), and Figs. 4(a-d)). Due to the shock wave generated by the nanosecond laser pulses, the heat is absorbed by the surrounding material of the micro-holes. As a result, the ablated area was stressed, which in turn caused the formation of halos around the micro-holes. In addition, shock wave is responsible for the ejection of melted molecules from the ablated area edges [6]. However, shockwave lasts for maximum several nanoseconds and the atoms of the sample material are relaxed and ready to receive the next pulse where the arrival time of the next pulse is much longer [19]. Therefore, the configuration of heat affected zone around the multi-beam engraved micro-holes is almost similar to the heat affected zone around the singlebeam engraved micro-holes.

The proposed three-beam technology represented significant improvement of the OTR through the OPP films in compared to the single-beam technology assisted OPP films [3]. The OTR can be increased by increasing the pulse energy and the number of irradiated laser pulses. In addition, the focal depth of the laser beams has significant impact on the number of required overlapped pulses for fabricating breathable films. Use of a galvanometer scanner in place of objective lens might be useful in decreasing the required number of laser pulses.

In single-beam laser writing technique, when a laser beam is scanned on a sample surface, periodic holes are formed where each pulse is irradiated. To increase the width and depth of the micro-holes, we require applying more pulses in the same spot. If we want to irradiate three pulses as an example in each spot, we need to scan the laser beam three times and consequently processing time is increased and throughput is decreased. In addition, when a laser beam is applied three times in each irradiated spot, the laser pulses may hit the samples slightly different locations due to the lack of synchronization between the laser beam and the translation stage. Compared to single-beam technology proposed in [3-6], processing time has been decreased at least three times in our proposed three-beam technology if all other experimental parameters remain same. Therefore, we can triple the throughput during fabricating breathable films by sequential application of three laser beams in the same spot. However, the overall processing time is solely dependent on the scanning speed and the pulse repetition rate of the laser source. During our experiment, it required only 6 minutes to fabricate breathable OPP films over an area of $3 \mathrm{~cm} \times 3 \mathrm{~cm}$ using three pulses in each spot. Currently, laser sources are available with very high pulse repetition rate even up to several $\mathrm{MHz}$. As a result, the translation stage can operate at a speed of several meters per second, which in turn can significantly reduce the processing time and increase the throughput during fabricating breathable films. The throughput can further be increased by increasing the number of split beams, which can be achieved by using higher order diffractive beam splitters such as $4 \times 4,2 \times 6$, or even higher order.

\section{Conclusion}

In this paper, we proposed a novel technique of fabricating breathable films by patterning periodic micro-holes' array on the surface of OPP films using diffractive multibeams of an ultraviolet pulsed nanosecond laser. By using a $1 \times 3$ diffractive beam splitter, we generated three parallel diffractive beams, which were irradiated sequentially on top of the OPP films' surface. Accordingly, three pulses are 
irradiated in each spot that increases the diameter and depth of the micro-holes formed on the OPP films. The depth and diameter of the micro-holes showed increasing trend with the increase of pulse energy of the laser beams. Multiple pulses engraved OPP films showed excellent oxygen transfer rate, i.e., breathability where the breathability can be increased by increasing the number of incident laser pulses in each irradiated spot. By increasing the number of parallel diffractive beams, we can increase the throughput of breathable films manufacturing. Our proposed technique is easy, flexible, and fast to be considered for commercial applications.

\section{Acknowledgements}

Our research work was supported by GIST Research Institute (GRI) grant funded by GIST, Republic of Korea in 2020. In addition, the research work was supported by the National Research Foundation of Korea (NRF) grant funded by the Korean Government (MSIT) (NRF2017M2A8A4017221).

\section{References}

[1] P.C. Wu, G. Jones, C. Shelly, and B. Woelfil: J. Eng. Fiber. Fabr., 2, (2007) 49.

[2] C.M. Adams, R.J. Downs, and K.J. Mcdaniels: U. S. Patent 8784968 (2014)

[3] I.B. Sohn, Y.C. Noh, S.C. Choi, D.K. Ko, J. Lee, and Y.J. Choi: Appl. Surf. Sci., 254, (2008) 4919.

[4] I.B. Sohn, Y.C. Noh, Y.S. Kim, D.K. Ko, and J. Lee: J. Opt. Soc. Korea, 12, (2008) 38.

[5] H.K. Choi, I.B. Sohn, Y.C. Noh, Y.J. Choi, and I.G. Chang: J. Korean Soc. Prec. Eng., 30, (2013) 409.

[6] Z. Wang, Z. Du, J.K.Y. Chan, S.H. Teoh, E.S. Thian, and M. Hong: ACS Biomater. Sci. Eng., 2015, (2015) 1239.
[7] T. Sakai, N. Nedyalkov, and M. Obara: Appl. Phys., 40, (2007) 7485.

[8] T. Sakai, Y. Tanaka, Y. Nishizawa, M. Terakawa, and M. Obara: Appl. Phys. A, 99, (2010) 39.

[9] Z.B. Wang, W. Guo, A. Pena, D.J. Whitehead, B.S. Lukýanchuk, L. Li, Z. Liu, Y. Zhou, and M.H. Hong: Opt. Express, 16, (2008) 19706.

[10] J.I. Kato, N. Takeyasu, and Y. Adachi: Appl. Phys. Lett., 86, (2005) 044102.

[11]Z. Kuang, D. Liu, W. Perrie, S. Edwardson, M. Sharp, E. Fearon, G. Dearden, and K. Watkins: Appl. Surf. Sci., 255 (2009) 6582.

[12]Z. Kuang, W. Perrie, J. Leach, M. Sharp, S.P. Edwardson, M. Padgett, G. Dearden, K.G. Watkins: Appl. Surf. Sci., 255, (2008) 2284.

[13]Z. Kuang, W. Perrie, D. Liu, S. Edwardson, J. Chengh, G. Dearden, and K. Watkins: Appl. Surf. Sci., 255, (2009) 9040.

[14] M. Hirano, K. Kawamura, and H. Hosono: Appl. Surf. Sci., 197-198, (2002) 688.

[15]Z. Guo, S. Qu, Y. Han, and S. Liu: Opt. Comm., 280, (2007) 23.

[16] T. Kondo, S. Juodkazis, V. Mizeikis, H. Misawa, and S. Matsuo: Opt. Express, 14, (2006) 7943.

[17] R. Zhou, S. Lin, Y. Ding, H. Yang, K.O.Y. Keng, and M. Hong: Opto-Electron. Adv., 1, (2018) 180014.

[18] M.S. Ahsan, Y.G. Kim, and M.S. Lee: J. Non Cryst. Solids, 357, (2011) 851.

[19] A. Vogel, S. Busch, and U. Parlitz: J. Accoust. Soc. Am., 100, (1996) 148.

[20] M.S. Ahsan and M.S. Lee: "Femtosecond Laser Processing of Materials" (Lambert Academic Publishing, Germany, 2013).

(Received: June 17, 2020, Accepted: July 26, 2020) 